\title{
On Designing an Active Tail for Legged Robots: Simplifying Control via Decoupling of Control Objectives
}

\author{
Steve W. Heim ${ }^{1,2}$, Mostafa Ajallooeian ${ }^{2}$, Peter Eckert ${ }^{2}$, Massimo Vespignani ${ }^{2}$ and Auke Ijspeert $^{2}$
}

\begin{abstract}
This work explores the possible roles of active tails for steady-state legged-locomotion. A series of simple models are proposed which capture the dynamics of an idealized running system with an active tail. The models suggest that the control objectives of injecting energy into the system and stabilizing body-pitch can be effectively decoupled via proper tail design: a long, light tail. Thus the overall control problem can be simplified, using the tail exclusively to stabilize body-pitch: this effectively relaxes the constraints on the leg-actuators, allowing them to be recruited specifically for adding energy into the system. We show in simulation that models with long-light tails are better able to reject perturbations to body-pitch than short-heavy tails with the same moment of inertia. Further, we present the results of a one degree-of-freedom tail mounted on the open-loop controlled quadruped robot Cheetah-Cub. Our results show that an active tail can greatly improve both forward velocity and reduce body-pitch per stride, while adding minimal complexity. Further, the results validate the long-light tail design: shorter, heavier tails are much more sensitive to configuration and control parameter changes than longer and lighter tails with the same moment of inertia.
\end{abstract}

\section{INTRODUCTION}

Due to the very nature of the field, advances in legged robotics are very frequently bio-inspired, both in mechanical design (Altendorfer et al., 2001; Ananthanarayanan et al., 2012; Hutter et al., 2012) as well as control strategies ( Ajallooeian et al., 2013; Geyer and Herr, 2010; Ijspeert, 2008). Dynamic legged locomotion is a challenging task, with nonlinear hybrid dynamics. Although conventional engineering approaches are proving themselves at solving these problems (Boaventura et al., 2012; Mordatch et al., 2010), studying and understanding how animals solve them often leads to great simplifications (Holmes et al., 2006). In many cases animals achieve locomotion over steady terrain through a combination of periodic feed-forward control signals, generated via central pattern generators (CPGs) (Ijspeert, 2008), simple decentralized feedback in the form of reflexes (Geyer and Herr, 2010; Owaki et al., 2013), as well as mechanical design with natural dynamics displaying good stability and efficiency properties (Pfeifer and Iida, 2005; Rummel and Seyfarth, 2008). This last concept is often referred to as morphological computation or mechanical intelligence, and can be crucial to designing a versatile, high-performance legged robot (Bhounsule et al., 2012; Hauser et al., 2011; Spröwitz et al., 2013). With this in mind, we turn our attention to tails. In an aquatic environment, the importance of the tail

\footnotetext{
${ }^{1}$ Dept. of Mechanical and Process Engineering, ETHZ, Switzerland.

2 Biorobotics Laboratory (BIOROB), EPFL, Switzerland.

Corresponding Author: Steve W. Heim heim. steve@gmail.com

This is an extended version of the paper presented at CLAWAR 2015.
}

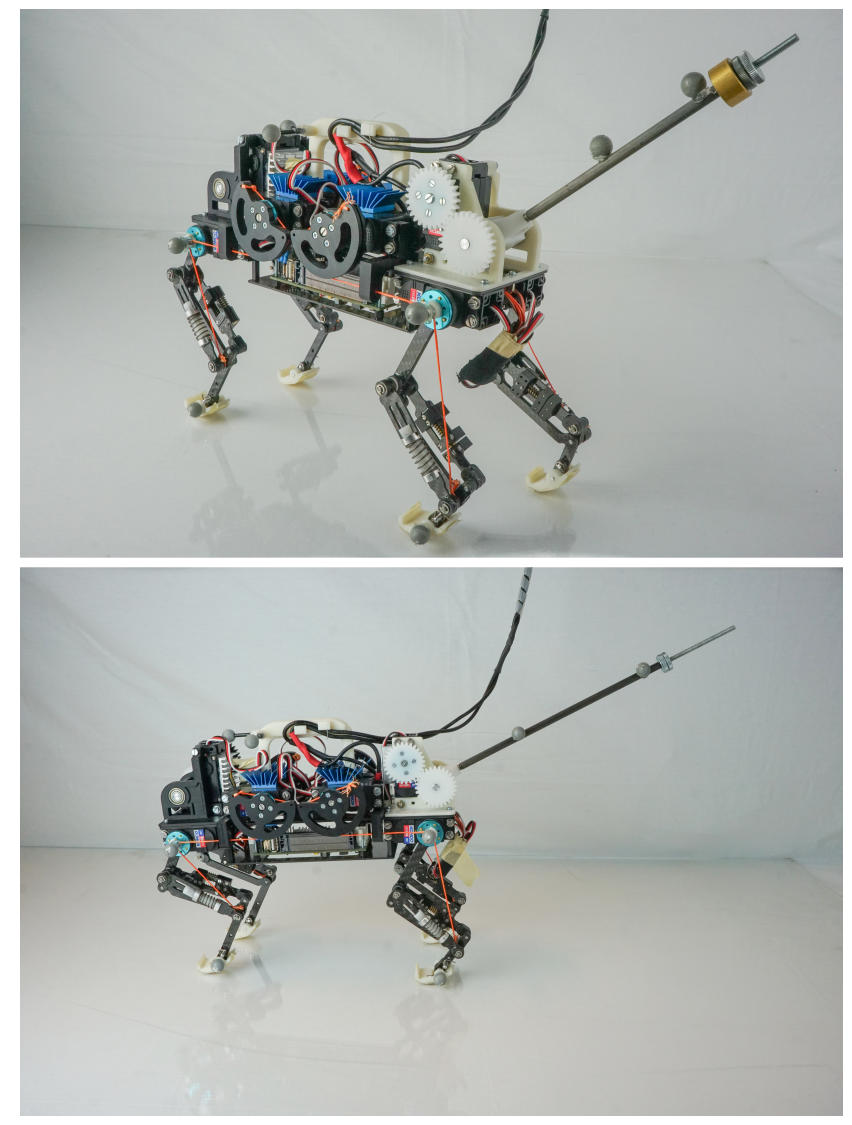

Fig. 1: Experiments were conducted using a newly built version of the Cheetah-Cub robot (Spröwitz et al., 2013), with an added 1 degree of freedom tail module. Shown are two different tails, a short-heavy (above) and long-light (below) tail. Both tails have the same moment of inertia around the tail axle. For the tail parameters see TABLE I. Brightness in the photographs was digitally enhanced.

for propulsion has been explored both in biology as well as in robotics (Bainbridge, 1958; Lauder and Drucker, 2004; Triantafyllou et al., 1993; Weihs, 1989). Despite this, the role of tails for locomotion in legged terrestrial vertebrates is still unclear. Although most terrestrial vertebrates possess a tail, having evolved from their aquatic predecessors, in most cases it has been adapted for purposes other than locomotion, especially among larger mammals (Hickman, 1979). Nonetheless there are some notable exceptions: kangaroo rats and pocket mice have been observed performing precise aerial maneuvering using their tail (Bartholomew and Caswell, 1951; Hickman, 1979). Geckos and other lizards have also been shown to use their tails extensively for aerial posture-control, particularly for controlling body-pitch 
(Jusufi et al., 2008; Libby et al., 2012) and the concept has been demonstrated to be useful in robotics as well (Johnson et al., 2012; Libby et al., 2012; Liu et al., 2014). Among larger terrestrial mammals, cheetahs appear to use their rather large tails to aid in cornering at high speeds, a concept that has been recently explored in robotics (Briggs et al., 2012; Patel and Braae, 2013). Snow leopards also appear to use their tails for stability while hunting on near vertical cliffs, though no studies have as of yet demonstrated this. Kangaroos, another large mammal with a relatively large tail (Alexander and Vernon, 1975), also appear to use their tails during hopping for controlling posture. In each of these last three cases, there are no conclusive biological experiments that prove the use of the tail to the best of our knowledge. It is however interesting to note that each case represents a highly specialized form of locomotion, ranging from high acceleration and speed (cheetah) to rough terrain (snow leopard) to efficiency (kangaroos). We believe that further understanding of these extreme examples can greatly aid in designing specialized robots, as well as understanding biology. As in Libby et al., 2012 we limit our study to the sagittal plane; we focus however on steady-state locomotion (i.e. constant speed), taking into consideration the dynamics of both flight and stance phases. We approach the problem through model-analysis and simulation of a simplified, idealized system by which we extract a basic design principle; we then validate this principle by applying it to a more complex system in hardware, using the Cheetah-Cub robot (Spröwitz et al., 2013), shown in Fig. 1. We thus show that, for the same moment of inertia (MoI), a longer and lighter tail design allows body-pitch control to be decoupled from other dynamics, simplifying control and adding robustness to the system.

\section{HYPOTHESIS}

We define a tail as an appendage which alters the dynamics of the system, without directly applying contact forces, i.e. without touching the ground. In this manner, when a kangaroo (or robot) uses its tail for support while standing or shuffling (O'Connor et al., 2014) we consider it as an additional leg instead of a tail. Conversely, any limb capable of inertial manipulation could in theory occupy the same role, as discussed also in (Johnson et al., 2012). In this study we only discuss tails, though the concepts can be directly transferred to other appendages. Other appendages such as the head or legs in swing-phase are typically subject to stricter constraints due to their various functional roles: for example, it is generally desirable to keep the head steady, both to protect the brain as well as to obtain better visual sensory input. We hypothesize that a proper tail design allows for a much simpler control strategy, by decoupling the control problem. This is similar in concept to Raibert's original control scheme (M. H. Raibert, 1986) in which hoppingheight, forward-velocity and body-pitch were each controlled independently by means of leg-thrust, landing angle-of-attack and hip-torque during the stance phase, respectively. This decoupled control scheme is not only simple but also very effective. However, the control objective of stabilizing bodypitch is partly simplified by designing the body to have a high moment of inertia and having the hip-joint located near the body center of gravity (CoG). More importantly, hip-torque is made available exclusively for body-pitch stabilization during the stance phase by having a prismatic leg, such that leg-thrust is largely independent of hip-torque. Most leggedrobots however use rotary motors driving articulated legs with rotary joints (Boaventura et al., 2012; Hutter et al., 2012; M. Raibert et al., 2008; Seok et al., 2013), in which case hip-torque becomes important to generating leg-thrust. In biology also, several studies indicate that hip-torque plays a key role in generating leg-thrust (Tan and Wilson, 2011). Indeed, the coupling between energy-input and body-pitch can be the overriding constraint to acceleration (Williams et al., 2009). For the sake of simplicity, we group the two control objectives of hopping-height and forward-velocity into a single objective, that of energy-input. We hypothesize that a properly designed active tail can allow for an alternative path to decoupling the body-pitch and energy-input control objectives: if the tail actuator has negligible impact on the energy-input, it can be used exclusively for controlling body-pitch in a simple control loop. Similarly, if the leg actuators have a negligible impact on body-pitch then they can be freely optimized for the energy-input control objective without having to consider body-pitch. This leads to two separate, simpler control schemes. In order for these two control objectives to be effectively separated, the dynamic coupling between the tail actuator input and the energyinput control objective should be minimized, or ideally, fully decoupled. The same is true for the leg actuators and the body-pitch control objective. This decoupling implies a specific tail design. We examine the requirements for this decoupling through a simple, idealized models.

\section{MODEL}

In order to understand the use of an active tail, we examine a simple idealized model which still captures the salient dynamics of the system, as in (Full and Koditschek, 1999). We base our model on the Spring-Loaded Inverted Pendulum (SLIP) model (Blickhan, 1989; Hutter et al., 2010). We extend it as shown in Fig. 2, to include three rigid bodies (body, leg and tail). We derive the full equations of motion (EoM) for both stance and flight phase using Lagrange's second method and obtain

$$
M_{(q)} \ddot{q}=H_{(q, \dot{q})}+B_{(q)} u_{(t)}+J_{c(q)}^{\top} \lambda_{G R F}
$$

where $q$ is the vector of generalized coordinates, $\dot{q}$ and $\ddot{q}$ are the corresponding velocities and accelerations respectively, $M_{(q)}$ is the mass matrix, $H_{(q, \dot{q})}$ contains differentiable forces, $B_{(q)}$ is the control matrix, $u_{(t)}$ is the vector of control inputs, $\lambda_{G R F}$ is the ground-reaction force (GRF) in cartesiancoordinates and $J_{c(q)}$ is the contact Jacobian of the foot, 
which we use to project $\lambda_{G R F}$ into the generalized coordinates. The vector of degrees of freedom (DoF) is $q_{(t)}=$ $\left(x, y, \varphi, y_{F}, \varphi_{H}, \varphi_{T}\right)^{\top}$ (see Fig. 2). Note that our system has the full 6 DoF during flight-phase, however during stancephase we assume no slipping of the foot, and therefore the vector of minimal coordinates is reduced to 4 DoF. Instead of switching between two separate minimal vectors $q$, we keep the same vector $q$ for both flight- and stance-phases but add two constraint equations during the stance-phase in the form of the ground-reaction forces $\lambda_{G R F}$, which enforce the no-slip assumption. This allows a more intuitive analysis of the effect of each control input in $u_{(t)}$. For more details on how this constraint is calculated, see Remy et al., 2011.

For the analysis, we then solve (1) explicitly, yielding

$$
\begin{aligned}
& \ddot{q}=M_{(q)}^{-1}\left(H_{(q, \dot{q})}+B_{(q)} u_{(t)}+J_{c(q)}^{\top} \lambda_{G R F}\right) \\
& \ddot{q}=\bar{H}_{(q, \dot{q})}+\bar{B}_{(q)} u_{(t)}
\end{aligned}
$$

where we have rewritten the solution and separated all terms that multiply a control input into $\bar{B}_{(q)}$ and the rest into $\bar{H}_{(q, \dot{q})}$. In the simplest case, we use a flywheel instead of a tail (see $b$ ) in Fig. 2), i.e. a tail with moment of inertia (MoI) but no length or mass, and with both tail and hip joints located directly on the body center of gravity. The matrices $\bar{H}_{(q, \dot{q})}$ and $\bar{B}_{(q)}$ as well as the control vector $u_{(t)}$ are shown in (4) and (5).

$$
\begin{aligned}
& \bar{H}=\left(\begin{array}{c}
\frac{J_{F} \cos \left(\varphi_{H}\right)\left(g \sin \left(\varphi_{H}\right)-2 \dot{\varphi}_{H} \dot{y}_{F}\right)}{J_{F}+m_{B} y_{F}^{2}} \\
\frac{g\left(J_{F} \cos 2 \varphi_{H}+J_{F}+2 m_{B} y_{F}^{2}\right)+4 J_{F} \dot{\varphi}_{H} \sin \left(\varphi_{H}\right) \dot{y}_{F}}{2\left(J_{F}+m_{B} y_{F}^{2}\right)} \\
0 \\
y_{F} \dot{\varphi}_{H}^{2}-g \cos \left(\varphi_{H}\right) \\
\frac{m_{B} y_{F}\left(g \sin \left(\varphi_{H}\right)-2 \dot{\varphi}_{H} \dot{y}_{F}\right)}{J_{F}+m_{B} y_{F}^{2}} \\
0
\end{array}\right) \\
& \bar{B}_{(q)} u_{(t)}=\left(\begin{array}{ccc}
\frac{\sin \left(\varphi_{H}\right)}{m_{B}} & \frac{y_{F} \cos \left(\varphi_{H}\right)}{J_{F}+m_{B} y_{F}^{2}} & 0 \\
-\frac{\cos \left(\varphi_{H}\right)}{m_{B}} & \frac{y_{F} \sin \left(\varphi_{H}\right)}{J_{F}+m_{B} y_{F}^{2}} & 0 \\
0 & -\frac{1}{J_{B}} & -\frac{1}{J_{B}} \\
\frac{1}{m_{B}} & 0 & 0 \\
0 & \frac{1}{J_{F}+m_{B} y_{F}^{2}} & 0 \\
0 & 0 & \frac{1}{J_{T}}
\end{array}\right)\left(\begin{array}{c}
\tau_{F} \\
\tau_{H} \\
\tau_{T}
\end{array}\right)
\end{aligned}
$$

Here, $m$ and $J$ refer to mass and moment of inertia, $g$ is gravity and the sub-indices ${ }_{B}, F$ and $T$ indicate the body, foot and tail respectively. If we examine the explicit EoM for this case, we see that the flywheel DoF has no influence at all on the rest of the system dynamics: it is fully decoupled. This changes drastically if the joint is displaced from the body center of gravity $(\mathrm{CoG})$ or full tail with length and mass is used instead of a flywheel. More importantly, in the control matrix $\bar{B}_{(q)}$ we see that the flywheel torque $\tau_{T}$ only affects the tail-rotation $\varphi_{T}$ and the body-pitch $\varphi$ but not the other body DoF $x$ and $y$. Indeed the only coupling between body-pitch $\varphi$ and body-translation $x$ or $y$ DoF is through the hip-torque $\tau_{H}$. This lends itself well to a very simple control structure: the $x$ and $y$ coordinates of the body carry most of the kinetic and potential energy during locomotion, and in particular the $y$ DoF oscillates between high kinetic and high potential energy. In other words, these are the two coordinates with which energy-input to maintain locomotion is associated. The body-pitch DoF $\varphi$ on the other hand, should ideally contain as little energy as possible: the angular momentum of the body has to integrate to zero, otherwise the body will start tumbling. Thus, any variations of energy-content, i.e. angular momentum, in the body imply an equal amount of positive and negative work, which could otherwise be put to better use to displace the body. As long as the coupling between body pitch and translation is not too strong, the coupling can effectively be ignored and treated as a disturbance, and a simpler single-input single-output control loop can be applied to body-pitch, with the tail-torque $\tau_{T}$ as control input. In order to do this, the tail design should approximate the dynamics of a flywheel.

This is the key advantage of using a highly-idealized model such as the one shown in $b$ ) in Fig. 2: because the dynamics are much simpler, it is possible to calculate the explicit EoM symbolically as shown in Eq. 2 and 3 and analyze them with relative ease. This allows for easy abstraction of key principles. We do not go into the details of design analysis here, though the reader may refer to Heim, 2014; suffice it to say the tail should be able to carry significant angular momentum while carrying minimal linear momentum. In other words, while keeping the MoI of the tail constant, a long and light tail is preferable to a short and heavy tail.

\section{SIMULATION RESULTS}

We use the MATLAB framework developed by C. Remy (Remy et al., 2011), with minor modifications. The entire framework is released open-source with several examples, including the models presented here, and can be found at https://bitbucket.org/ramlab/ optimal_gait_creation_framework.

The framework disposes of gradient-based optimization tools to synthesize locally-optimal, open-loop control trajectories for a periodic gait, which require a relatively good initial guess. Since the models presented in section III are very sensitive to even small changes in parameters, finding initial guesses becomes a tedious manual operation. For this reason we proceed in two steps: quasi-periodic initial guesses are first generated using a particle-swarm optimization (Van Den Kieboom et al., 2014) framework running on a cluster of 80 nodes, and gradient-based optimization is then applied to these results. To generate the initial-guesses we simply maximize distance traveled before falling, while limiting torques to avoid giant leaps. This simple method reliably produces solutions which are close enough to being both periodic as well as locally optimal that they can be used as an initial guess. We then perform Floquet analysis on the return map (Guckenheimer and Holmes, 1983) of these 


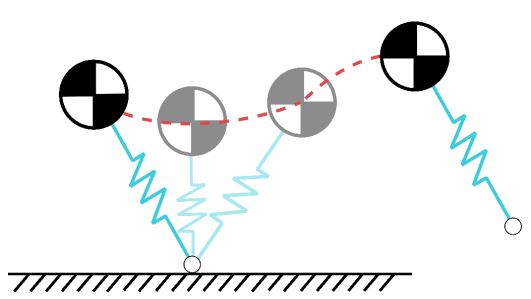

a)

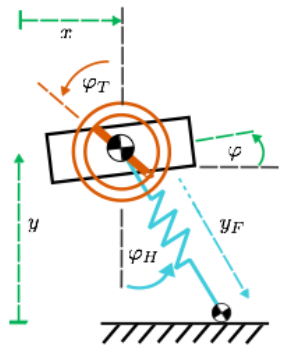

b)

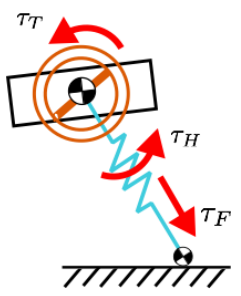

c)

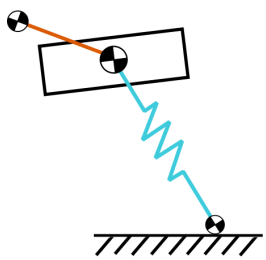

d)

Fig. 2: a) Different key instances of the general Spring-Loaded Inverted Pendulum (SLIP) model are shown, from left to right: touch-down, maximum leg-compression, lift-off and flight-phase apex. Note that, differently from our models, the original SLIP model is not actuated but at the same time energy-conservative i.e. there are no energy losses. Also, the body-pitch degree of freedom (DoF) is not modeled and the hip-angle is simply reset for each new hop.

b) Our simplest model uses a flywheel, which represents an idealized tail with moment of inertia (MoI) but no mass. The dynamics and physical parameters of all DoF are modeled; these DoF are shown in $b$ ).

c) Here the control inputs are depicted: a force $\tau_{F}$ acting on the leg-extension DoF $y_{F}$, a torque $\tau_{H}$ acting on the hip-rotation DoF $\varphi_{H}$ and a torque $\tau_{T}$ acting on the tail-rotation $\operatorname{DoF} \varphi_{T}$.

d) In the final model we use an actual tail with not only MoI but also length, therefore an off-center mass. The DoF and control inputs remain the same as shown in $b$ ) and $c$ ).

solutions to limit-cycle stability, as well as generate a closedloop controller which stabilizes a first-order linearization of the return-map as in Remy et al., 2011. We compare the response of systems with different tail-designs to body-pitch velocity perturbations, with both open-loop and closed-loop controllers. We keep the MoI of the tail around the tail-joint constant, while adjusting the length and mass accordingly. The results are shown in Fig. 3. As we expect due to the increased coupling and resultingly more complex control problem, the simple closed-loop controller implemented has progressively more trouble rejecting body-pitch perturbations as the flywheel becomes a tail, and the tail becomes shorter and heavier, despite maintaining the same MoI and thus the same inertial effectiveness (Johnson et al., 2012). These results match the predictions we make in section III.

\section{HARDWARE RESULTS}

A 1-DoF rigid tail was built and mounted on the CheetahCub robot (Spröwitz et al., 2013). Cheetah-Cub is a small quadruped with two actuated DoF per leg, hip and kneeflexion, slightly smaller and lighter than a house-cat. The Cheetah-Cub features compliant pantographic legs which make it robust to impacts, and more importantly allow for stable and fast open-loop locomotion: feed-forward positioncontrol inputs are fed directly to the servo-motors, generated from a simple CPG network. Stability is provided by the mechanical morphology of the robot and indeed for the trotting gait there is a very wide set of parameters of the CPG which result in stable locomotion. This allows the CPG to be tuned for performance, i.e. forward speed, without worrying about stability issues. Though previous research with the Cheetah-Cub has focused on a trotting gait, we chose a bounding gait, i.e. with the fore pair and hind pair of legs moving in unison as can be seen in Fig. 6. We chose bounding since body-pitching (and therefore the effect of the tail on body-pitch control) is more pronounced and relevant in this gait. The CPG nodes of the hips are tuned to be inphase left-right, and anti-phase fore-hind. The tail CPG node is connected directly to the hind-hip nodes in anti-phase. For more details, see Heim, 2014. Other connection schemes between the tail and leg CPG nodes were briefly explored, but no differences of great interest arose. Our tails, shown in Fig. 4, are made with a hollow carbon-fiber tube, weighted at the end with aluminum and brass weights. This design makes it not only easy to adjust the tail parameters but is also easily modeled as a massless rod and a point-mass.

For the presented comparison, we use two tails with almost the same moment of inertia (MoI): a short-heavy tail with length of $12.8 \mathrm{~cm}$ (from tail-joint to $\mathrm{CoG}$ ), mass of 53 grams and MoI of $8.68 \mathrm{~kg} \mathrm{~cm}^{2}$, and a long-light tail with length $16.8 \mathrm{~cm}$, mass of 31 grams and MoI of $8.75 \mathrm{~kg} \mathrm{~cm}^{2}$. For reference, the entire robot without the tail weighs $1.25 \mathrm{~kg}$ and measures $21 \mathrm{~cm}$ from hip to hip. The key parameter is the MoI. For values which are too low, tail performance is limited by the range of tail-sweep; if it is too high, performance is limited by maximum motor torque. Within a reasonable range of $\mathrm{MoI}$ around the one used, changes in MoI can be compensated by tuning tail amplitude, resulting in little overall impact on performance. In our experiments, we hand-tune the CPG-parameters to find a gait which represents a local optimum in terms of forwardvelocity for both the short-heavy tail as well as the long-light tail. We then change the values of individual CPG-parameters and observe the effect. In general, we observe two situations: either the effect is the same using either tail, as is the case for changing the phase-lag between the tail and hind-hip CPG nodes (forward velocity drops in much the same way for both tails), or the parameter change has a much greater effect on the performance of the robot with short-heavy tail than it does when equipped with a long-light tail. This is the case, 


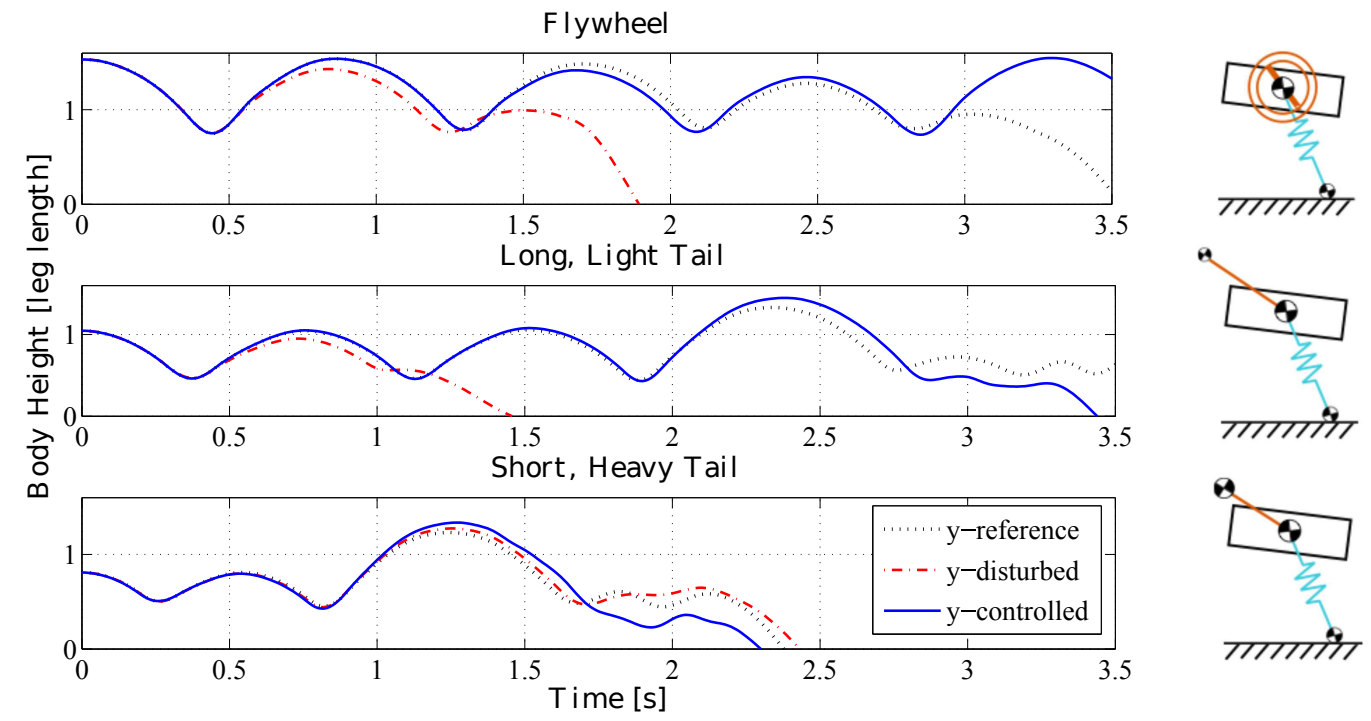

Fig. 3: We compare the responses of several models to a small perturbation to the body-pitch velocity: plotted are the open-loop reference with no perturbation (black, dashed), open-loop response to perturbation (red, dot-dashed) and closed-loop response (blue, solid). For more details on the closed-loop controller, see Remy et al., 2011. Note that none of the limit-cycles are stable and therefore any small perturbation, whether physical or numerical, will cause the system to diverge. In the three cases, the moment of inertia of the tails remains the same: the first represents a flywheel and as such has a MoI but no off-center mass, i.e. the length of the tail is zero. In the second two cases, the tail mass and length are scaled in such as way as to ensure the total MoI remains the same: the shorter tail is half the length, but four times the weight. As we progress from a the flywheel model, with no 'tail mass', to tails which are progressively heavier, the effectiveness of the closed-loop controller diminishes: in the short-heavy tail model, it has essentially no effect on stability, visualized here as steps-to-falling.

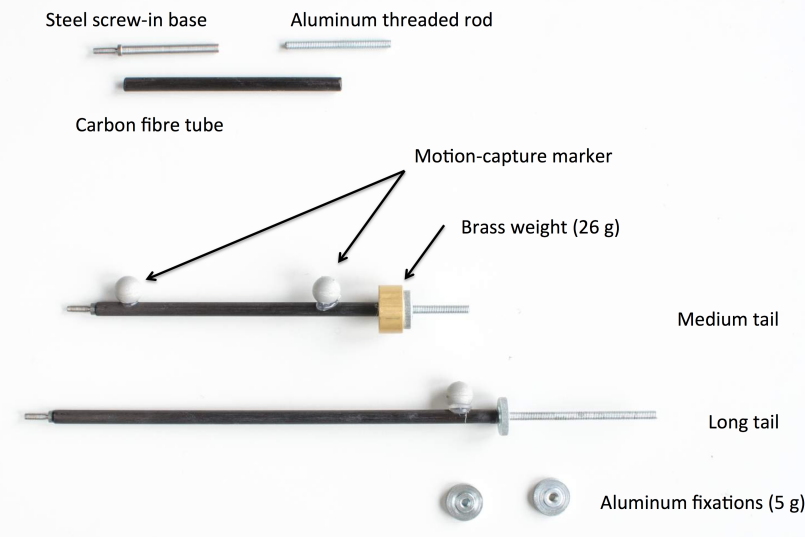

Fig. 4: The tail was designed in a very simple manner which allows weight to be easily adjusted, and many versions of the tail with different lengths can be cheaply and quickly constructed. The tails can be exchanged on the robot within seconds with the simple screw-in base. Note, the steel base was necessary due to the high moments sustained by the weighted tail. Brightness in the photographs was digitally enhanced.

for example, when changing the tail-offset or tail amplitude. We show in Fig. 5 and TABLE I the experimental results for changing the tail-offset, which is the parameter with the most pronounced difference between tail configurations. For comparison, we also show the performance of the robot with a rigid, passive tail, i.e. where the tail is kept at a fixed angle of 30 degrees with respect to the body. We use this as a comparison instead of comparing with no tail at all in order to keep the total MoI of the robot as similar as possible across all three configurations. Further, without a tail, all the remaining CPG parameters need to be retuned to achieve a meaningful gait. The best bounding gaits found without a tail at all were similar in performance to the presented case with a passive tail. In the trials shown, all CPG node parameters are kept constant across each configuration, except for those specified: in the tail CPG node the tail-offset, i.e. the midpoint of the tail oscillations, is changed between trials $a$ ) and $c$ ) compared to trials $b$ ) and $d)$. In trial $e$ ) 'passive-tail' the tail CPG-node is disconnected from the rest and has an amplitude of 0, i.e. it keeps a fixed position. We find that performance is very similar between the two tails when perfectly tuned, as in cases $a$ ) and $c$ ). However, when equipped with a short-heavy tail, changing the tail-offset by just 30 degrees has a notably larger effect on performance, with forward velocity decreasing $7.35 \%$. With the long-light tail, performance remains essentially the same. Further, standard deviation of body-pitching is and remains much lower when using the long-light tail regardless of tail-offset, implying a steadier, smoother gait. Considering that the entire robot weighs $1.25 \mathrm{~kg}$ and even the heavy tail represent only $4.4 \%$ of the total body-mass, such a large effect on performance is impressive. We find that these results match our predictions: since both tails have almost the same MoI they are about equally effective at stabilizing body-pitch, however the longer lighter tail is less sensitive to configuration changes, making the robot potentially more versatile in a larger portion of state-space. 


\begin{tabular}{|c|c|c|c|c|c|c|c|c|}
\hline \multirow{4}{*}{$\begin{array}{l}\text { Tail Parameters } \\
\text { Short-heavy tail } \\
\text { length: } 12.8[\mathrm{~cm}] \\
\text { mass: } 53[\mathrm{~g}] \\
\text { MoI: } 8.68\left[\mathrm{~kg} \mathrm{~cm}^{2}\right]\end{array}$} & \multirow{3}{*}{$\begin{array}{l}\text { Plot } \\
\text { a) }\end{array}$} & \multirow{3}{*}{$\begin{array}{l}\text { Tail Position [degrees] } \\
\text { offset: } 35\end{array}$} & \multicolumn{3}{|c|}{ Forward Velocity $\left[\frac{m}{s}\right]$} & \multicolumn{3}{|c|}{ Body Pitching per Stride [degrees] } \\
\hline & & & mean & $\begin{array}{l}\text { standard } \\
\text { deviation }\end{array}$ & runs & mean & $\begin{array}{l}\text { standard } \\
\text { deviation }\end{array}$ & $\begin{array}{l}\text { number of } \\
\text { strides }\end{array}$ \\
\hline & & & 0.558 & 0.012 & 4 & 8.7 & 1.6 & 32 \\
\hline & b) & offset: 65 & 0.517 & 0.036 & 8 & 6.6 & 2.1 & 59 \\
\hline \multirow{2}{*}{$\begin{array}{l}\text { Long-light tail } \\
\text { length: } 16.8[\mathrm{~cm}] \\
\text { mass: } 31[\mathrm{~g}] \\
\text { MoI: } 8.75\left[\mathrm{~kg} \mathrm{~cm}^{2}\right]\end{array}$} & c) & offset: 35 & 0.554 & 0.023 & 8 & 5.7 & 1.0 & 58 \\
\hline & d) & offset: 65 & 0.556 & 0.011 & 8 & 4.6 & 1.0 & 63 \\
\hline $\begin{array}{l}\text { Passive Tail } \\
\text { length: } 12.8[\mathrm{~cm}] \\
\text { mass: } 53[\mathrm{~g}] \\
\text { MoI: } 8.68\left[\mathrm{~kg} \mathrm{~cm}^{2}\right]\end{array}$ & $e)$ & offset: 35 & 0.479 & 0.034 & 6 & 8.5 & 3.5 & 53 \\
\hline
\end{tabular}

TABLE I: We tested the effect of the active, open-loop tail on the performance of Cheetah-Cub using a bounding gait. A sample of the dataset is shown here, and visualized in Fig. 5. The performance metrics we use are forward velocity and body-pitching; the sample size and standard deviation are also shown for each metric. In the 'passive tail' configuration the tail is kept at a fixed angle with respect to the body; we chose to use this configuration for comparison instead of 'no tail' to keep the total MoI across each trial as similar as possible. Without the active tail, we were not able to find control parameters which resulted in forward velocity of over $0.5\left[\frac{\mathrm{m}}{\mathrm{s}}\right]$. More importantly, the robot is much less stable laterally and frequently falls on its side, and also frequently requires many steps with close to 0 velocity (it simply runs in place with its feet slipping on the ground) before settling into a proper gait. This settling phase was trimmed when calculating the results, and trials which ended in a fall were discarded.

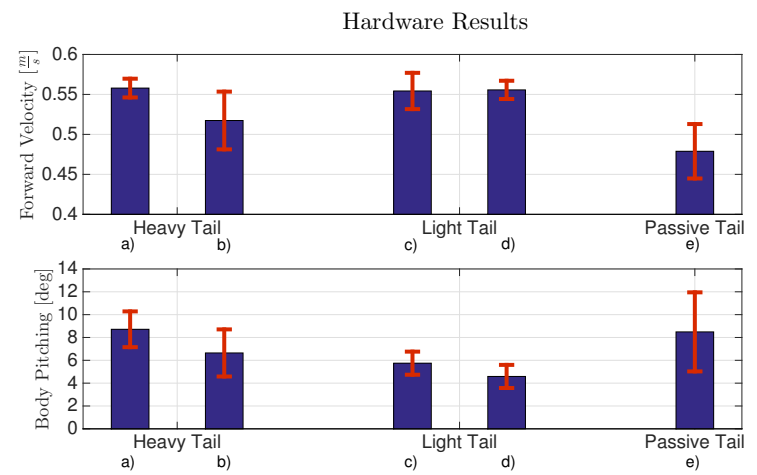

Fig. 5: The results shown in TABLE I are visualized here. Of greatest interest is the comparison of the two examples shown with active-tails: they have almost the same moment of inertia, but the shorter tail is $71.0 \%$ heavier. With the short-heavy tail the robot is more sensitive to changes in many of the control parameters. We show here one of the critical parameters: the tail-offset with respect to the body. Performance barely changes with the long-light tail, while with the short-heavy tail forward velocity drops $7.4 \%$.

\section{CONCLUSIONS AND OUTLOOK}

Using a simple, idealized mathematical model we have extracted a design principle which shows that a long and light tail-design, approximating the dynamics of a flywheel, results in a much lower degree of coupling between the energy-input and body-pitch control objectives. Thus using a long and light tail or flywheel to control body-pitch relaxes the constraints on the leg-actuators, potentially allowing more effective actuation for energy-input. We verified this principle in a simple single-leg simulation and demonstrated it's applicability to more complex systems through hardware experiments with the Cheetah-Cub quadruped robot. From our analysis, a flywheel is most effective in decoupling and

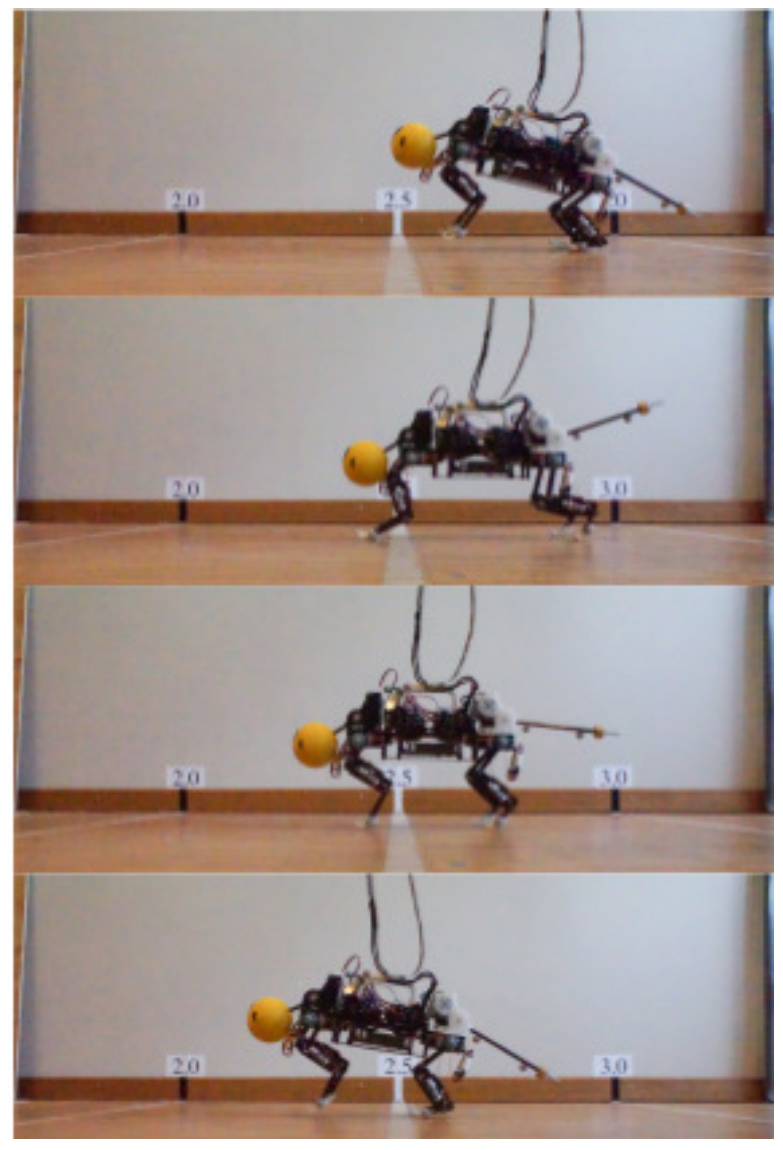

Fig. 6: Snapshots taken at 30 FPS over a stride-cycle of the CheetahCub bounding with an active, long-light tail. From the snapshots the in-phase motion of left and right legs of the bounding gait can be seen clearly. The two cables provide power as well as communication via ethernet. All computation is handled on-board the robot. The head is a light foam ball which was attached for aesthetics, and does not affect the dynamics in a relevant way. 
thus simplifying the control problem. A tail can however be more suitable due to a number of design constraints as discussed in Briggs et al., 2012 and Libby et al., 2012. Most notably, a tail can have a higher MoI-to-mass ratio and is generally easier to integrate into a design or existing platform due to the dimensional requirements of a flywheel. As a next step, we are working on a more in-depth stability analysis in simulation to identify the effect of an active tail on regions of attraction, in terms of both control and design parameters. A comparison with a full closed-loop controller in hardware would also be of great interest, especially on different platforms, across different scales and in different situations, as explored in Johnson et al., 2012. Though we focused on the advantage of a simplified control problem by decoupling body-pitch and energy-input control objectives, actually exploiting the coupling is another approach which should also be taken into consideration. De and Koditschek, 2015 show that the tail can be used specifically for energyinput instead of body-pitch control. Indeed, we suspect kangaroos may be able to get away with a relatively large and heavy tail despite their size (Alexander and Vernon, 1975, McGowan et al., 2008) due to an evolutionary pressure favoring high efficiency due to large distances between foraging spots, while not requiring high locomotor versatility in terms of agility, acceleration or speed due to a relatively flat, easy to cope with habitat as well as a general scarcity of natural predators. In this situation, perhaps the potential energetic benefits of the coupled-dynamics outweigh the necessity for simpler control.

\section{ACKNOWLEDGMENT}

We would like to thank all the members of the BioRob lab for constructive conversations and feedback, as well as interns and students who helped carry out tests. In particular we thank François Longchamp and Daniel Chapuis for their contribution in the hardware development, Daniel Renjewski for advice on stability analysis, Jesse van den Kieboom for his help in using the PSO framework. We would also like to thank various friends and colleagues who proofread the manuscript and provided valuable feedback.

Funding was received from the European Community's Seventh Framework Program (FP7/2007-2013 - Challenge 2-Cognitive Systems, Interaction, Robotics; grant agreement number 248311 (AMARSi)) and from the Swiss National Science Foundation through the National Centre of Competence in Research Robotics.

\section{REFERENCES}

Ajallooeian, M. et al. (2013). "Modular control of limit cycle locomotion over unperceived rough terrain". Intelligent Robots and Systems (IROS), 2013 IEEE/RSJ International Conference on. Ieee, pp. 3390-3397.

Alexander, R. M. and A. Vernon (1975). "The mechanics of hopping by kangaroos (Macropodidae)". Journal of Zoology 177.2, pp. 265-303.

Altendorfer, R. et al. (2001). "RHex: a biologically inspired hexapod runner”. Autonomous Robots 11.3, pp. 207-213.
Ananthanarayanan, A. et al. (2012). "Towards a bio-inspired leg design for high-speed running". Bioinspiration \& biomimetics 7.4, p. 046005.

Bainbridge, R. (1958). "The speed of swimming of fish as related to size and to the frequency and amplitude of the tail beat". Journal of experimental biology 35.1, pp. 109-133.

Bartholomew, G. A. and H. H. Caswell (1951). "Locomotion in kangaroo rats and its adaptive significance". Journal of Mammalogy, pp. 155-169.

Bhounsule, J. et al. (2012). "Design and control of Ranger: an energy-efficient, dynamic walking robot". Proc. CLAWAR, pp. 441-448.

Blickhan, R. (1989). "The spring-mass model for running and hopping". Journal of biomechanics 22.11, pp. 1217-1227.

Boaventura, T. et al. (2012). "Dynamic torque control of a hydraulic quadruped robot". Robotics and Automation (ICRA), 2012 IEEE International Conference on. IEEE, pp. 1889-1894.

Briggs, R. et al. (2012). "Tails in biomimetic design: Analysis, simulation, and experiment". Intelligent Robots and Systems (IROS), 2012 IEEE/RSJ International Conference on. IEEE, pp. 1473-1480.

De, A. and D. Koditschek (2015). "Parallel Composition of Templates for Tail-Energized Planar Hopping". Robotics and Automation (ICRA), 2013 IEEE International Conference on.

Full, R. J. and D. Koditschek (1999). "Templates and anchors: neuromechanical hypotheses of legged locomotion on land". Journal of Experimental Biology 202.23, pp. 3325-3332.

Geyer, H. and H. Herr (2010). "A muscle-reflex model that encodes principles of legged mechanics produces human walking dynamics and muscle activities". Neural Systems and Rehabilitation Engineering, IEEE Transactions on 18.3.

Guckenheimer, J. and P. Holmes (1983). Nonlinear oscillations, dynamical systems, and bifurcations of vector fields. Vol. 42. Springer Science \& Business Media.

Hauser, H. et al. (2011). "Towards a theoretical foundation for morphological computation with compliant bodies". Biological cybernetics 105.5-6, pp. 355-370.

Heim, S. (2014). "Design and Control of an Active Tail for Robot Locomotion". Master Thesis. EPF Lausanne and ETH Zurich. URL: http: / / biorob.epfl.ch/students/heim.

Hickman, G. C. (1979). "The mammalian tail: a review of functions". Mammal review 9.4, pp. 143-157.

Holmes, P. et al. (2006). "The dynamics of legged locomotion: Models, analyses, and challenges". Siam Review 48.2.

Hutter, M. et al. (2010). "Slip running with an articulated robotic leg". Intelligent Robots and Systems (IROS), 2010 IEEE/RSJ International Conference on. IEEE, pp. 4934-4939.

Hutter, M. et al. (2012). StarlETH: A compliant quadrupedal robot for fast, efficient, and versatile locomotion. World Scientific.

Ijspeert, A. J. (2008). "Central pattern generators for locomotion control in animals and robots: a review". Neural Networks 21.4, pp. 642-653.

Johnson, A. et al. (2012). "Tail Assisted Dynamic Self Righting". Proceedings of the Fifteenth International Conference on Climbing and Walking Robots, pp. 611-620.

Jusufi, A. et al. (2008). "Active tails enhance arboreal acrobatics in geckos". Proceedings of the National Academy of Sciences 105.11, pp. 4215-4219.

Lauder, G. V. and E. G. Drucker (2004). "Morphology and experimental hydrodynamics of fish fin control surfaces". Oceanic Engineering, IEEE Journal of 29.3, pp. 556-571.

Libby, T. et al. (2012). "Tail-assisted pitch control in lizards, robots and dinosaurs". Nature 481.7380, pp. 181-184.

Liu, G.-H. et al. (2014). "A Bio-Inspired Hopping Kangaroo Robot with an Active Tail". Journal of Bionic Engineering 11.4, pp. 541-555.

McGowan, C. P. et al. (2008). "Differential design for hopping in two species of wallabies". Comparative Biochemistry and 
Physiology Part A: Molecular \& Integrative Physiology 150.2, pp. 151-158.

Mordatch, I. et al. (2010). "Robust physics-based locomotion using low-dimensional planning". ACM Transactions on Graphics (TOG) 29.4, p. 71.

O'Connor, S. M. et al. (2014). “The kangaroo's tail propels and powers pentapedal locomotion”. Biology letters 10.7.

Owaki, D. et al. (2013). "Simple robot suggests physical interlimb communication is essential for quadruped walking". Journal of The Royal Society Interface 10.78, p. 20120669.

Patel, A. and M. Braae (2013). "Rapid turning at high-speed: Inspirations from the cheetah's tail". Intelligent Robots and Systems (IROS), 2013 IEEE/RSJ International Conference on. IEEE, pp. 5506-5511.

Pfeifer, R. and F. Iida (2005). "Morphological computation: Connecting body, brain and environment". Japanese Scientific Monthly 58.2, pp. 48-54.

Raibert, M. H. (1986). Legged robots that balance. MIT press.

Raibert, M. et al. (2008). "Bigdog, the rough-terrain quadruped robot". Proceedings of the 17th World Congress. Vol. 17. 1, pp. 10822-10825.

Remy, C. D. et al. (2011). "A matlab framework for efficient gait creation". Intelligent Robots and Systems (IROS), 2011 IEEE/RSJ International Conference on. IEEE, pp. 190-196.

Rummel, J. and A. Seyfarth (2008). "Stable running with segmented legs". The International Journal of Robotics Research 27.8, pp. 919-934.

Seok, S. et al. (2013). "Design principles for highly efficient quadrupeds and implementation on the MIT Cheetah robot". Robotics and Automation (ICRA), 2013 IEEE International Conference on. IEEE, pp. 3307-3312.

Spröwitz, A. et al. (2013). "Towards dynamic trot gait locomotion: Design, control, and experiments with Cheetah-cub, a compliant quadruped robot". The International Journal of Robotics Research 32.8, pp. 932-950.

Tan, H. and A. M. Wilson (2011). "Grip and limb force limits to turning performance in competition horses". Proceedings of the Royal Society B: Biological Sciences 278.1715, pp. 2105-2111.

Triantafyllou, G. S. et al. (1993). "Optimal thrust development in oscillating foils with application to fish propulsion". Journal of Fluids and Structures 7.2, pp. 205-224.

Van Den Kieboom, J. et al. (2014). "Meta Morphic Particle Swarm Optimization". Nature Inspired Cooperative Strategies for Optimization (NICSO 2013). Springer, pp. 231-244.

Weihs, D. (1989). "Design features and mechanics of axial locomotion in fish". American Zoologist 29.1, pp. 151-160.

Williams, S. B. et al. (2009). "Pitch then power: limitations to acceleration in quadrupeds". Biology letters. 